\title{
CORIORRETINOPATÍA SEROSA CENTRAL COMO MANIFESTACIÓN EXTRADIGESTIVA DE INFECCIÓN GÁSTRICA POR HELICOBACTER PYLORI
}

\author{
CENTRAL SEROUS CHORIORETINOPATHY AS AN \\ EXTRADIGESTIVE MANIFESTATION OF HELICOBACTER \\ PYLORI GASTRIC INFECTION
}

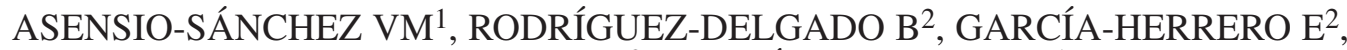 \\ CABO-VAQUERA V², GARCÍA-LOYGORRI C ${ }^{1}$
}

\section{RESUMEN}

Objetivo: La infección gástrica por Helicobacter pylori (HP) es considerada como un factor de riesgo importante en la patología arterial oclusiva. Actualmente se piensa que la coriorretinopatía serosa central (CSC) es una enfermedad por oclusión multifocal en la coriocapilar. El objeto de este estudio es valorar una posible relación patogénica entre la infección gástrica por HP y la CSC.

Material y método: Se estudió un grupo de 16 pacientes con CSC y 20 controles. El estudio de la infección por HP se realizó con el test respiratorio de la urea-13C (TRU). El diagnóstico clínico de CSC se confirmó con biomicroscopía posterior y angiografía fluoresceínica.

Resultados: De los 16 pacientes CSC, 11 (68,75\%) fueron varones y $5(31,25 \%)$ mujeres con una edad media de 46,3 años. La infección por HP fue positiva en 11 pacientes $(68,75 \%)$ y negativa en 5 $(31,25 \%)$. Los hombres fueron HP positivo (HP+) en el $72,7 \%$ de los casos frente a las mujeres que fueron $\mathrm{HP}+$ en el $60 \%$. La diferencia en prevalencia de HP entre el grupo-CSC $(68,75 \%)$ y el grupo-con-

\section{ABSTRACT}

Objective: Helicobacter pylori (HP) gastric infection has been implicated as an important factor in occlusive arterial pathology. Nowadays, it is suspected that central serous chorioretinopathy (CSC) is due to a multifocal vascular occlusive disease of the choriocapillaris. The aim of this study was to determine the relation between gastric HP infection and CSC.

Materials and methods: We evaluated a group of 16 patients with CSC and 20 controls. HP infection was assessed by the 13C-urea breath test (UBT). Clinical CSC diagnosis was confirmed by fundus biomicroscopy and fluorescein angiography.

Results: Out of 16 patients with CSC, 11 (68.75\%) were males and $5(31.25 \%)$ females, with a mean age of 46.3 years. HP infection was positive in 11 patients $(68.75 \%)$ and negative in $5(31.25 \%)$. Men were HP-positive (HP+) in $72.7 \%$ of cases, compared to women who were HP+ in $60 \%$ of cases. The difference in prevalence of HP between the CSCgroup (68.75\%) and the control-group (30\%) was found to be statistically significant $(\mathrm{p}<0.05)$. HP+

\footnotetext{
Recibido: 6/7/06. Aceptado: 15/2/08.

Hospital General. Medina del Campo. Valladolid. España.

1 Doctor en Medicina.

2 Licenciado en Medicina.

Correspondencia:

V.M. Asensio Sánchez

Hospital General Servicio Castellano-Leonés de Salud

Servicio de Oftalmología

Medina del Campo (Valladolid)

España

E-mail: vasensio@hmdc.sacyl.es
} 
trol $(30 \%)$ fue significativa $(\mathrm{p}<0,05)$. Los pacientes $\mathrm{HP}+$ tenían más dolores gástricos que los pacientes HP negativo (HP-) (72,73\% vs 20\%).

Conclusiones: Estos resultados indican una posible asociación estadística entre la infección gástrica por Helicobacter pylori y la coriorretinopatía serosa central, pudiendo considerar la infección por HP como un posible factor de riesgo en los pacientes CSC.

Palabras clave: CSC, Helicobacter pylori, test respiratorio de la urea, infección gástrica, manifestación extradigestiva, factor de riesgo. patients had more gastric pain than HP negative (HP-) patients $(72.73 \%$ vs $20 \%)$.

Conclusions: These results indicate a possible statistical association between Helicobacter pylori gastric infection and CSC. HP should thus be considered a risk factor in CSC patients. (Arch Soc Esp Oftalmol 2008; 83: 177-182).

Key words: CSC, Helicobacter pylori, urea breath test, gastric infection, extradigestive manifestation, risk factor.

\section{INTRODUCCIÓN}

La coriorretinopatía serosa central (CSC) clásicamente se define como un desprendimiento seroso neurosensorial de la retina de origen desconocido que afecta a la mácula generalmente durante episodios de estrés emocional y típicamente a varones jóvenes entre 25 y 45 años (1). La mayoría de los casos tienen una recuperación funcional espontánea, pero a veces en los pacientes de mayor edad se puede cronificar con signos de descompensación del epitelio pigmentario (EP) retiniano y pérdida grave de visión $(1,2)$. La etiopatogenia de la CSC es desconocida $(1,2)$ aunque siempre se ha relacionado con personalidades tipo A, personas ansiosas y estresadas, considerando que en la fase aguda se produce una liberación de corticoides (cortisol) y adrenalina. La adrenalina puede causar daño en la coriocapilar e incrementar su permeabilidad. El cortisol puede potenciar la acción vasoconstrictora de las catecolaminas. La inhibición de la síntesis de colágeno por el cortisol puede producir adelgazamiento de la pared capilar resultando en un aumento de su fragilidad favoreciendo la permeabilidad de la coriocapilar (1-3). El Helicobacter pylori (HP) es una bacteria gram negativa gástrica de aspecto espiral que se asocia a múltiples patologías digestivas y extradigestivas (4-10). HP no atrajo el interés de los oftalmólogos hasta recientemente (11-13). Este trabajo tiene por objeto establecer una posible relación patogénica entre la infección gástrica por Helicobacter pylori y la coriorretinopatía serosa central.

\section{SUJETOS, MATERIAL Y MÉTODOS}

Este estudio fue aprobado por la Comisión de Investigación y Docencia de nuestro hospital. Los pacientes procedían de la consulta de retina. Se estudiaron 36 personas (16 pacientes y 20 controles) entre los años 2002 y 2006. En la tabla I se presentan las características demográficas de ambos grupos. Los criterios de inclusión para los pacientes (grupo-CSC) fueron los siguientes:

- Aceptar participar en el estudio con firma del consentimiento informado.

- No haber sido diagnosticado previamente de infección por HP.

- No haber sido tratado tres meses previos al estudio con antibióticos, corticoides, citrato de sildenafilo o fármacos simpático-miméticos. En las mujeres en edad fértil prueba de embarazo negativo.

- Fondo de ojo: Desprendimiento seroso retiniano asociado o no a un desprendimiento seroso del epitelio pigmentario.

- Angiografía fluoresceínica: Uno o varios puntos de fuga a nivel del epitelio pigmentario retiniano.

Los criterios de inclusión para los controles (grupo-control) fueron:

- Aceptar participar en el estudio con firma del consentimiento informado.

- Ser cónyuge o hermanos de pacientes del grupo anterior.

- No tener historia pasada o presente de CSC.

- No haber sido diagnosticado previamente de infección por HP.

Tabla I. Características demográficas

\begin{tabular}{lcc}
\hline & Grupo control & Grupo CSC \\
\hline N. & 20 & 16 \\
Sexo & $13(65 \%)$ varones & $11(68,75 \%)$ varones \\
Edad: & 50,2 D.E. 12,4 & 46,3 D.E.13,2 \\
\hline \hline
\end{tabular}

CSC: Coriorretinopatía serosa central; N. ${ }^{\circ}$ : número. 
- No haber sido tratado tres meses previos al estudio con antibióticos, corticoides, citrato de sildenafilo o fármacos simpático-miméticos. En las mujeres en edad fértil prueba de embarazo negativo.

Los criterios de exclusión fueron:

- No aceptar las condiciones del estudio.

- Imposibilidad para realizar el seguimiento.

- Embarazo, lactancia y puerperio.

- Tratamiento con corticoides, antibióticos, simpático-miméticos, citrato de sildenafilo y vitaminas en el presente o tres meses antes del estudio.

- Colagenopatías o trasplantados.

La valoración del dolor gástrico se realizó con:

- Escala descriptiva simple o de valoración verbal (VRS): clasifica en no dolor, suave, moderado, mucho e insoportable.

- Escala de Andersen: medición del dolor desde el punto de vista dinámico y lo cuantifica según se describe de forma espontánea, en reposo, con movimientos ligeros o con la tos.

Se realizó una exploración oftalmológica completa que incluyó angiografía fluoresceínica. El estudio del HP se hizo con un método no invasivo, el test repiratorio de la urea (TRU): a cada paciente se le dio para ingerir una solución de $100 \mathrm{mg}$ de urea $\mathrm{C}^{13}$ asociada a ácido cítrico. Cuando HP coloniza la mucosa gástrica, la ureasa bacteriana metaboliza la urea en NH3 (amoniaco) y en CO2 (dióxido de carbono) que se elimina por el aire expirado y con un espectrómetro de masas se cuantifica el $\mathrm{C}^{13}$. Nuestro laboratorio pone el valor de corte en $2,5 \mathrm{C}^{13} \% 00\left(\mathrm{HP}+>2,5 \mathrm{C}^{13} \% 00\right)$.

Para comparar las variables cualitativas entre los pacientes y el grupo control se utilizó el chi cuadrado y la t de Student para las variables cuantitativas.

\section{RESULTADOS}

En la tabla II se muestra la comparación entre los pacientes y el grupo control con respecto a HP. De los 16 pacientes con CSC, once $(68,75 \%)$ fueron positivos para HP y cinco casos $(31,25 \%)$ fueron negativos. En el grupo control, seis (30\%) fueron positivos para HP y 14 casos $(70 \%)$ fueron negativos. En la tabla III se muestra el porcentaje de HP por sexos. En el grupo-CSC los hombres fueron HP positivo en el $72,73 \%$ de los casos frente a las mujeres que fueron positivas en el $60 \%$. En el grupoControl el $30,77 \%$ de los hombres fueron positivos frente al 28,57\% de las mujeres. Cuando se realiza
Tabla II. Comparación Helicobacter pylori

\begin{tabular}{lcr}
\hline & Grupo control & Grupo CSC \\
\hline $\mathrm{HP}+$ & $6(30 \%)$ & $11(68,75 \%)$ \\
$\mathrm{HP}-$ & $14(70 \%)$ & $5(31,25 \%)$ \\
\hline \hline
\end{tabular}

HP: Helicobacter pylori; CSC: Coriorretinopatía serosa central.

un análisis estratificado de la muestra según el sexo, se observa que el HP se asocia a una menor edad de diagnóstico de CSC en el grupo de hombres $(\mathrm{HP}+=$ 32,2 DE 4,1; HP- = 39,8 DE 5,7: p<0,001). La comparación de la proporción de casos positivos a HP del grupo control (30\%) y del grupo-CSC $(68,75 \%)$ fue significativa $(\mathrm{p}<0,05)$.

Los pacientes positivos para HP tenían más dolores gástricos (ocho de los once, $72,73 \%$ ) que los pacientes negativos para HP (uno de los cinco, 20\%).

\section{DISCUSIÓN}

La coriorretinopatía serosa central es un desprendimiento seroso macular que afecta típicamente a varones jóvenes entre 25 y 45 años que habitualmente tiene una resolución espontánea con un buen pronóstico visual (1). En un pequeño porcentaje de pacientes se desarrolla una enfermedad crónica o progresiva con descompensación del EP y pérdida severa de visión $(1,2)$. Actualmente la etiopatogenia de la CSC no es completamente conocida y hasta el momento no hay tratamiento efectivo. Con la CSC se han relacionado tóxicos como el tabaco, fármacos como los corticoides, simpaticomiméticos, antibióticos y más recientemente citrato de sildenafilo, el embarazo, diversos procesos sistémicos como hipertensión arterial, colagenopatías, asma, y enfermedades oftalmológicas como la retinopatía pigmentaria (14-16). Se ha especulado mucho sobre el origen psicosomático de la CSC al asociarse con personalidades tipo A $(3,14)$. Iijima $\mathrm{H}$ et al (17) en

Tabla III. Comparación Helicobacter pylori

\begin{tabular}{lcc}
\hline & Grupo control & Grupo CSC \\
\hline $\mathrm{HP}+$ & $\mathrm{V}=4(30,77 \%)$ & $\mathrm{V}=8(72,73 \%)$ \\
$\mathrm{HP}-$ & $\mathrm{M}=2(28,57 \%)$ & $\mathrm{M}=3(60 \%)$ \\
& $\mathrm{V}=9(69,23 \%)$ & $\mathrm{V}=3(27,27 \%)$ \\
& $\mathrm{M}=5(71,43 \%)$ & $\mathrm{M}=2(40 \%)$ \\
\hline \hline
\end{tabular}

HP: Helicobacter pylori; CSC: Coriorretinopatía serosa central; V: Varones; M: Mujer. 
pacientes con CSC describieron un aumento del inhibidor 1 del activador tisular del plasminógeno. A nivel de la coriocapilar se producirían zonas de oclusión con isquemia en el epitelio pigmentario por lo que la CSC sería un proceso microvascular que afectaría de forma generalizada al EP, dato que se corresponde con los hallazgos clínicos. Recientemente se ha documentado que la CSC pudiera ser una manifestación extradigestiva del HP(18-21). El $\mathrm{HP}$ es una bacteria microaerófila gram negativa con forma espiral que coloniza el estómago del $50 \%$ de los humanos. Su presencia se asocia a la inflamación de la mucosa gástrica motivada por la infiltración de células inflamatorias. Antes de su descubrimiento en 1984, se pensaba que el estrés y algunos factores relacionados con el estilo de vida eran los responsables de la úlcera péptica (22). Sin embargo, hoy se sabe que la bacteria es responsable de al menos el $90 \%$ de las úlceras duodenales y el $80 \%$ de las gástricas. En este trabajo se ha estudiado la relación entre HP y CSC en un grupo de pacientes frente a un grupo control. El grupo control al estar formado esencialmente por cónyuges de los enfermos con CSC, minimiza el efecto de las variables medioambientales y económico-sociales que pueden influir en la colonización por HP (22). El TRU, utilizado en este trabajo, es el método no invasivo fundamental para la detección de la infección activa por HP con una sensibilidad del 90\%, los límites de esta prueba vienen determinados por su coste y por la necesidad de un alto nivel de equipamiento (23). En la serie que se presenta la CSC afecta más a hombres $(68,75 \%)$ que a mujeres $(23,1 \%)$, con una edad media de 46,3 años. La infección por HP fue positiva en 11 pacientes $(68,75 \%)$ y negativa en $5(31,25 \%)$. Los hombres fueron HP positivo en el $72,73 \%$ de los casos frente a las mujeres que fueron HP positivas en el $60 \%$, en concordancia con los resultados de otros trabajos que también mostraron una mayor prevalencia de infección por HP en varones que en mujeres en general, independientemente de la afectación ocular (24). La diferencia en prevalencia de HP entre el grupo-CSC $(68,75 \%)$ y el grupo-control $(30 \%)$ fue significativa $(\mathrm{p}<0,05)$. Es importante destacar que los pacientes $\mathrm{HP}+$ tenían más dolores gástricos que los pacientes HP(72,73\% vs $20 \%)$, lo cual clásicamente se ha asociado en la clínica práctica a la ansiedad y estrés de estos pacientes (3). En la valoración del dolor se utilizó la escala de valoración verbal (VRS), introducida por Keele en 1948. Representa el más bási- co abordaje para medir el dolor, y es útil para el investigador, debido a lo fácil de su aplicación. El paciente no suele encontrar gran dificultad en utilizar esta escala para indicar su nivel de dolor. La escala de Andersen realiza la medición del dolor desde el punto de vista dinámico. A la buena correlación que se ha observado con otras escalas hay que añadir su interés en el dolor agudo, dado que lo asocia a la disminución de la actividad física que provoca. No tenemos explicación de la asociación encontrada entre el HP y una manifestación más precoz de CSC en varones, aunque con seguridad influyen los niveles hormonales y de gastrina que controlan la secreción de las células parietales $(11,22)$. Mauget-Faysse $\mathrm{M}$ et al (18) en un estudio prospectivo con 16 pacientes-CSC del Sureste de Francia detectan en el 56,3\% infección por HP, porcentaje muy superior al $27,5 \%$ de la población control. Ahnoux-Zabsonre et al (19) en un estudio con 78 pacientes establecieron una asociación entre la infección por HP y CSC $(39,7 \%$ vs $25,4 \%$ del grupo control). Cotticelli L et al (25) comprobaron que la prevalencia de $\mathrm{HP}$ es significativamente mayor en pacientes con CSC que en el grupo control (78,2\% vs $43,5 \%$ ). De todos estos trabajos se puede considerar que HP puede representar un factor de riesgo en pacientes con CSC pudiendo estar implicado en su fisiopatología de la misma forma que afecta en patologías vasculares, cerebrales y dermatológicas: antígenos bacterianos serían homólogos a proteínas que expresa el cuerpo humano, como por ejemplo el endotelio vascular, produciéndose un ataque del sistema inmunitario a estas estructuras como la coriocapilar, con disminución del flujo sanguíneo y daño en el EP(11,20). ¿Por qué HP no siempre produce CSC?, quizás por las diferentes cepas de HP cada una con una genética diferente y por tanto con características bioquímicas y patogénicas distintas y a esto se asocian las citoquinas, prostaglandinas y leucotrienos $(12,22,26)$ que aumentan su concentración sanguínea en diferente concentración como consecuencia de la infección y que actuarían en los vasos coroideos. Otro factor limitante importante para el desarrollo CSC sería la susceptibilidad determinada genéticamente de las personas $(22,26)$. Los pacientes diagnosticados de CSC crónica y tratados con terapia fotodinámica pueden reducir los signos clínicos, como la permeabilidad coroidea. Sin embargo, la mejoría de la agudeza visual puede ser variable (27). Los antiangiogénicos podrían ser una opción terapeútica para la 
CSC crónica, sin embargo se precisan más estudios antes de generalizar su uso (28). Las limitaciones de este trabajo son las debidas al número de casos estudiados y a la falta de estudios genéticos-bacterianos, si ésto se confirmara con estudios multicéntricos, caso-control y randomizados, el tratamiento de la CSC sería esencialmente antimicrobiano al ser una manifestación extragástrica del Helicobacter pylori.

\section{BIBLIOGRAFÍA}

1. Lamkin JC, Singerman LJ, Addiego R. Laser treatment of macular diseases. In: Grossniklaus HE, Kincaid MC. Ophthalmology Clinics of North America. Macular Diseases. Philadelphia: Saunders; 1993; 317-337.

2. Gass JD, Little H. Bilateral bullous exudative retinal detachment complicating idiopathic central serous chorioretinopathy during systemic corticosteroid therapy. Ophthalmology 1995; 102: 737-747.

3. Keltikangas-Jarvinen L. The prevalence and construct validity of type A behaviour in patients with duodenal ulcers. Br J Med Psychol 1987; 60: 163-167.

4. Anto T, Goto Y, Maeda O, Watanabe O, Ishiguro K, Goto H. Causal role of Helicobacter pylori infection in gastric cancer. World J Gastroenterol 2006; 12: 181-186.

5. Gobbo AC, de Freitas M, Cury PM, Caetano A, Borim AA, Silva AE. Genetic alterations in benign lesions: chronic gastritis and gastric ulcer. World J Gastroenterol 2006; 12: 625-629.

6. Franceschi F, Roccarina D, Gasbarrini A. Extragastric manifestations of Helicobacter pylori. Minerva Med 2006; 97: 39-45.

7. Nilsson HO, Pietroiusti A, Gabrielli M, Zocco MA, Gasbarrini $G$, Gasbarrini A. Helicobacter pylori and extragastric diseases-other Helicobacters. Helicobacter 2005; 10: 54-65.

8. Gabrielli $M$, Santoliquido A, Cremonini F, Cicconi V, Candelli $M$, Serricchio $M$, et al. CagA-positive cytotoxic H. pylori strains as a link between plaque instability and atherosclerotic stroke. Eur Heart J 2004; 25: 64-68.

9. Gabrielli M, Pola P, Gasbarrini A. Helicobacter pylori, CagA-positive strains, and ischemic stroke. Stroke 2002; 33: 1453-1454.

10. Gasbarrini A, Franceschi F, Armuzzi A, Ojetti V, Candelli $M$, Torres ES, et al. Extradigestive manifestations of Helicobacter pylori gastric infection. Gut 1999; 45: I9I12.

11. Mindel JS, Rosenberg EW. Is Helicobacter pylori of interest to ophthalmologists? Ophthalmology 1997; 104: 1729-1730.

12. Franceschi F, Gasbarrini A, Fontana L. Role of Helicobacter pylori infection in ophthalmology. Ophthalmology 1998; 105: 1351-1352.
13. Sacca SC, Pascotto A, Venturino GM, Prigione G, Mastromarino A, Baldi F, et al. Prevalence and treatment of Helicobacter pylori in patients with blepharitis. Invest Ophthalmol Vis Sci 2006; 47: 501-508.

14. Haimovici R, Koh S, Gagnon DR, Lehrfeld T, Wellik S; Central Serous Chorioretinopathy Case-Control Study Group. Risk factors for central serous chorioretinopathy: a case-control study. Ophthalmology 2004; 111: 244:249.

15. Allibhai ZA, Gale JS, Sheidow TS. Central serous chorioretinopathy in a patient taking sildenafil citrate. Ophthalmic Surg Lasers Imaging 2004; 35: 165-167.

16. Cunningham ET Jr, Alfred PR, Irvine AR. Central serous chorioretinopathy in patients with systemic lupus erythematosus. Ophthalmology 1996; 103: 2081-2090.

17. Iijima H, Iida T, Murayama K, Imai M, Gohdo T. Plasminogen activator inhibitor 1 in central serous chorioretinopathy. Am J Ophthalmol 1999; 127: 477-478.

18. Mauget-Faysse M, Kodjikian L, Quaranta M, Ben Ezra D, Trepsat C, Mion F, et al. Helicobacter pylori in central serous chorioretinopathy and diffuse retinal epitheliopathy. Results of the first prospective pilot study. J Fr Ophtalmol 2002; 25: 1021-1025.

19. Ahnoux-Zabsonre A, Quaranta M, Mauget-Faysse $M$. Prevalence of Helicobacter pylori in central serous chorioretinopathy and diffuse retinal epitheliopathy: a complementary study. J Fr Ophtalmol 2004; 27: 1129-1133.

20. Giusti C. Association of Helicobacter pylori with central serous chorioretinopathy: hypotheses regarding pathogenesis. Med Hypotheses 2004; 63: 524-527.

21. Giusti C, Mauget-Faysse M. Helicobacter pylori and idiopathic central serous chorioretinopathy. Swiss Med Wkly 2004; 134:395-398.

22. Logan RP, Walker MM. ABC of the upper gastrointestinal tract: Epidemiology and diagnosis of Helicobacter pylori infection. BMJ 2001; 323: 920-922.

23. Hooton $C$, Keohane J, Clair J, Azam M, O'Mahony S, Crosbie $O$, et al. Comparison of three stool antigen assays with the $13 C$ - urea breath test for the primary diagnosis of Helicobacter pylori infection and monitoring treatment outcome. Eur J Gastroenterol Hepatol 2006; 18: 595-599.

24. de Martel C, Parsonnet J. Helicobacter pylori infection and gender: a meta-analysis of population-based prevalence surveys. Dig Dis Sci 20006; 51: 2292-2301.

25. Cotticelli L, Borrelli M, D’Alessio AC, Menzione M, Villani A, Piccolo $G$, et al. Central serous chorioretinopathy and Helicobacter pylori. Eur J Ophthalmol 2006; 16: 274278.

26. Logan RP, Berg DE. Genetic diversity of Helicobacter pylori. Lancet 1996; 348: 1462-1463.

27. Reche-Frutos J, Calvo-González C, Donate-López J, Sáenz-Francés-San-Baldomero F, Cerván-López, I, García-Feijoó J, y col. Photodynamic therapy in severe chronic central serous chorioretinopaty. Arch Soc Esp Oftalmol 2008; 83: 9-14.

28. Niegel MF, Schrage NF, Christmann $S$, Degenring RF. Intravitreal bevacizumab for chronic central serous chorioretinopathy. Ophthalmologe 2008 Jan 24. 